\title{
Thermal photons from gluon fusion with magnetic fields
}

\author{
Alejandro Ayala ${ }^{1,2, a}$, Jorge David Castaño-Yepes ${ }^{1, b}$, C. A. Dominguez ${ }^{2, c}$, and L. A. Hernández ${ }^{2, d}$ \\ ${ }^{1}$ Instituto de Ciencias Nucleares, Universidad Nacional Autónoma de México, Apartado Postal 70-543, Méx- \\ ico Distrito Federal 04510, Mexico. \\ ${ }^{2}$ Centre for Theoretical and Mathematical Physics, and Department of Physics, University of Cape Town, \\ Rondebosch 7700, South Africa
}

\begin{abstract}
We compute the production of thermal photons in relativistic heavy-ion collisions by gluon fusion in the presence of an intense magnetic field, and during the early stages of the reaction. This photon yield is an excess over calculations that do not consider magnetic field effects. We add this excess to recent hydrodynamic calculations that are close to describing the experimental transverse momentum distribution in RHIC and LHC. We then show that with reasonable values for the temperature, magnetic field strength, and strong coupling constant, our results provide a very good description of such excess. These results support the idea that the origin of at least some of the photon excess observed in heavy-ion experiments may arise from magnetic field induced processes.
\end{abstract}

The results from heavy-ion experiments carried out at the BNL Relativistic Heavy-Ion Collider (RHIC) and at the CERN Large Hadron Collider (LHC), show that a state of matter is formed where quarks and gluons are not confined to individual nucleons $[1,2]$. Non-central collisions produce magnetic fields with an intensity that at the beginning of the reaction is estimated to be as high as several times the mass of the pion squared [3-6], though their intensity fades out fast with time. At early times, it is also when the largest temperatures are achieved and when the soft dynamics is dominated by gluons.

A magnetic field makes it possible to produce photons from processes otherwise not allowed. Recent calculations [7-10] invoking the presence of these magnetic fields have been implemented to try to explain the experimentally measured excess [11] of thermal photons over models that describe well other low momentum observables.

A magnetic field naturally produces an asymmetry in the emission of electromagnetic radiation. Therefore, magnetic fields can be a source of not only an excess in the photon yield, but also of the puzzling large strength of the coefficient $v_{2}$ in the Fourier expansion of the azimuthal distribution. The latter has been found to be as large as that of pions [12]. Although some recently improved hydrodynamic $[13,14]$ and transport [15] calculations obtain a better agreement with ALICE and PHENIX measurements of low transverse momentum photons, this agreement is not yet complete [16]. Therefore, it remains important to quantify the fraction of the yield, and of the asymmetry arising from magnetic field effects, to better characterize the initial stages of heavy-ion reactions.

\footnotetext{
ae-mail: ayala@nucleares.unam.mx

be-mail: jorge.castano@correo.nucleares.unam.mx

ce-mail: cesareo.dominguez@uct.ac.za

de-mail: HRNLUI001@myuct.ac.za
} 
(a)

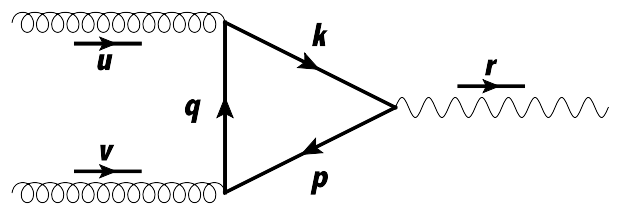

(b)

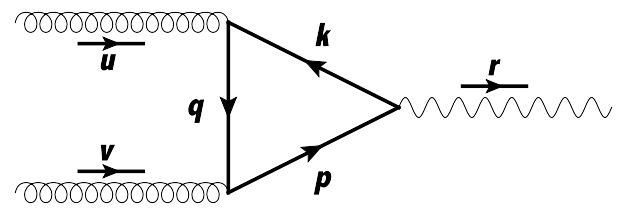

Figure 1. Feynman diagrams for the amplitude for photon production from gluon fusion. The thick lines in the loop represent the quark propagators in the presence of the magnetic field.

It is then natural to explore a mechanism where collisions of these gluons induce the emission of photons. In this work we outline the computation of the production of thermal photons from the perturbative fusion of gluons early in the collision. More details are provided in Ref. [17].

The amplitude for the process is depicted by the Feynman diagrams in Fig. 1, which also defines the kinematical variables. The thick loop lines represent the quark propagator in the presence of the magnetic field. In the absence of this field, the diagrams cancel each other. It is the presence of the field which makes it possible that both diagrams contribute with the same relative sign.

The fermion propagator in coordinate space cannot longer be written as a simple Fourier transform of a momentum propagator but instead it is written as [18]

$$
S\left(x, x^{\prime}\right)=\Phi\left(x, x^{\prime}\right) \int \frac{d^{4} p}{(2 \pi)^{4}} e^{-i p \cdot\left(x-x^{\prime}\right)} S(p),
$$

where

$$
\Phi\left(x, x^{\prime}\right)=\exp \left\{i q_{f} \int_{x^{\prime}}^{x} d \xi^{\mu}\left[A_{\mu}+\frac{1}{2} F_{\mu v}\left(\xi-x^{\prime}\right)^{v}\right]\right\},
$$

is called the phase factor, and $q_{f}$ is the absolute value of the quark charge. We consider the contribution of two light flavors, thus $q_{u}=2|e| / 3$ and $q_{d}=|e| / 3$. The propagator in momentum-space, $S(p)$, is given by

$$
\begin{aligned}
i S(p) & =\int_{0}^{\infty} \frac{d s}{\cos \left(q_{f} B s\right)} e^{i s\left(p_{\|}^{2}-p_{\perp} \frac{\tan \left(q_{f} B s\right)}{q_{f} B s}-m_{f}^{2}+i \epsilon\right)} \\
& \times\left[\left(\cos \left(q_{f} B s\right)+\gamma_{1} \gamma_{2} \sin \left(q_{f} B s\right)\right)\left(m_{f}+p_{\|}\right)-\frac{p_{\perp}}{\cos \left(q_{f} B s\right)}\right],
\end{aligned}
$$

where $m_{f}$ is the quark mass. We have chosen the homogeneous magnetic field to point in the $\hat{z}$ direction, namely $\boldsymbol{B}=B \hat{z}$. This configuration can be obtained from an external vector potential which we choose in the so called symmetric gauge $A^{\mu}=\frac{B}{2}(0,-y, x, 0)$. We have also defined $p_{\perp}^{\mu} \equiv$ $\left(0, p_{1}, p_{2}, 0\right), p_{\|}^{\mu} \equiv\left(p_{0}, 0,0, p_{3}\right), p_{\perp}^{2} \equiv p_{1}^{2}+p_{2}^{2}$ and $p_{\|}^{2} \equiv p_{0}^{2}-p_{3}^{2}$.

Since the two Feynman diagrams of Fig. 1 give the same contribution, we concentrate on the computation of the amplitude depicted in Fig. 1a which becomes

$$
\begin{aligned}
\mathcal{M}^{(a)} & =-\int d^{4} x \int d^{4} y \int d^{4} z \int \frac{d^{4} p}{(2 \pi)^{4}} \int \frac{d^{4} q}{(2 \pi)^{4}} \int \frac{d^{4} k}{(2 \pi)^{4}} e^{-i p \cdot(y-x)} e^{-i q \cdot(z-y)} e^{-i k \cdot(x-z)} e^{-i l \cdot z} e^{-i v \cdot y} e^{i r \cdot x} \\
& \times \operatorname{Tr}\left[i q_{f} \gamma_{\mu} i S(k) i g \gamma_{\alpha} t^{c} i S(q) i g \gamma_{v} t^{d} i S(p)\right] \epsilon^{* \mu}\left(\lambda_{r}\right) \Phi(x, y) \Phi(y, z) \Phi(z, x) \epsilon^{\alpha}\left(\lambda_{u}\right) \epsilon^{v}\left(\lambda_{v}\right)
\end{aligned}
$$


The product of phase factors can be written as

$$
\Phi(x, y) \Phi(y, z) \Phi(z, x)=e^{i \frac{q_{f} B}{2} \epsilon_{i j}(z-x)_{i}(x-y)_{j}},
$$

where we used the explicit form of $A^{\mu}$ which gives $F_{12}=-F_{21}=-B$, with the rest of the components of $F_{\mu \nu}$ vanishing, and $\epsilon_{i j}$ being the Levi-Civita symbol.

We use the fact that when the magnetic field is very intense, as compared to the other energy (squared) scales involved, the quark dynamics is dominated by the lowest Landau level (LLL). For the case of quarks that have not yet thermalized, this means that the magnetic field is taken to satisfy $e B \gg m_{f}^{2}$. For the LLL, the propagator in Eq. (3) can explicitly be written as

$$
i S(p)=2 i e^{-\frac{p_{\perp}^{2}}{q_{f} B}} \frac{\left(p_{\|}+m_{f}\right)}{p_{\|}^{2}-m_{f}^{2}} O_{\|}^{ \pm} .
$$

The operator $O_{\|}^{ \pm}=\left[1 \pm i \gamma_{1} \gamma_{2} \operatorname{sign}(e B)\right] / 2$ projects onto the longitudinal space. The positive (negative) sign corresponds to the case when the product $e B$ is positive (negative) and is to be used when the loop is made out of positively (negatively) charged quarks. Therefore the matrix element can be factorized into a product of transverse and longitudinal pieces, namely

$$
\begin{aligned}
\mathcal{M}^{(a)} & =(2 \pi)^{4} \delta^{4}(r-v-u) \mathcal{M}_{\perp}^{(a)} \mathcal{M}_{\|}^{(a)} \\
\mathcal{M}_{\perp}^{(a)} & =\left(\frac{4 \pi}{q_{f} B}\right)^{2} \int \frac{d^{2} p_{\perp}}{(2 \pi)^{2}} \int \frac{d^{2} q_{\perp}}{(2 \pi)^{2}} \int \frac{d^{2} k_{\perp}}{(2 \pi)^{2}} e^{-\frac{k_{\perp}^{2}}{q_{f} B}} e^{-\frac{q_{\perp}^{2}}{q_{f} B}} e^{-\frac{p_{\perp}^{2}}{q_{f} B}} \prod_{i, j=1,2} e^{i \frac{2}{q_{f} B} \epsilon_{i j}(q-k+u)_{i}(q-p-v)_{j}} \\
& =\left(\frac{q_{f} B}{12 \pi}\right) e^{-\frac{(u+v)_{\perp}^{2}}{3 q_{f} B}}, \\
\mathcal{M}_{\|}^{(a)} & =-8\left(\frac{q_{f} g^{2} \delta^{c d}}{2}\right) \int \frac{d^{2} p_{\|}}{(2 \pi)^{2}} \int \frac{d^{2} q_{\|}}{(2 \pi)^{2}} \int \frac{d^{2} k_{\|}}{(2 \pi)^{2}}(2 \pi)^{4} \delta^{2}\left[(q-k+u)_{\|}\right] \delta^{2}\left[(q-p-v)_{\|}\right] \\
& \times \frac{\operatorname{Tr}\left[\gamma_{\mu} k_{\|} O_{\|} \gamma_{\alpha} \phi_{\|} O_{\|} \gamma_{\nu} p_{\|} O_{\|}\right]}{k_{\|}^{2} q_{\|}^{2} p_{\|}^{2}} \epsilon^{\alpha}\left(\lambda_{u}\right) \epsilon^{v}\left(\lambda_{v}\right) \epsilon^{* \mu}\left(\lambda_{r}\right) .
\end{aligned}
$$

Since at the early stages of the collision gluons are far more abundant than quarks, we compute Eq. (9) under the assumption that quarks do not yet thermalize. Accordingly, we set $m_{f}=0$ since in the absence of thermal corrections, the light-quark vacuum masses are negligible. The trace in Eq. (9) contains the product of up to twelve gamma matrices. The resulting expression is long and involved. It is however easy to show that upon squaring and summing over polarizations, only a small piece survives so that the trace can be expressed as

$\operatorname{Tr}\left[\gamma_{\mu} k_{\|} O_{\|}^{ \pm} \gamma_{\alpha} \phi_{\|} O_{\|}^{ \pm} \gamma_{\nu} p_{\|} O_{\|}^{ \pm}\right] \rightarrow k_{\| v}\left(p_{\| \mu} q_{\| \alpha}-p_{\| \alpha} q_{\| \mu}\right)+k_{\| \mu}\left(p_{\| v} q_{\| \alpha}+p_{\| \alpha} q_{\| v}\right)+k_{\| \alpha}\left(p_{\| v} q_{\| \mu}-p_{\| \mu} q_{\| v}\right)$,

where the arrow indicates this to be the only contributing portion. Two of the integrations in Eq. (9) become straightforward using the delta-function restrictions. We choose those two as the integrals over $k_{\|}$and $q_{\|}$.

In order to make tractable the calculation of the longitudinal piece of the photon emission rate, Eq. (9), use is made of some simplifying assumptions, as is described in Ref. [17].

The final expression for the matrix element [Eqs. (7), (8) and (9)] squared, after adding the contribution from the Feynman diagram in Fig. 1b, summed over polarizations, becomes

$$
\sum_{\operatorname{pol}_{, f}}|\mathcal{M}|^{2}=\left(\frac{6256}{2187}\right)\left(\frac{\mathcal{V T}}{m_{g}^{2}}\right) \delta^{4}(r-v-u) \sum_{f}\left(q_{f} g^{2}\right)^{2}\left(q_{f} B\right)^{2} e^{-2 \frac{r_{\perp}^{2}}{3 q_{f} B}}
$$




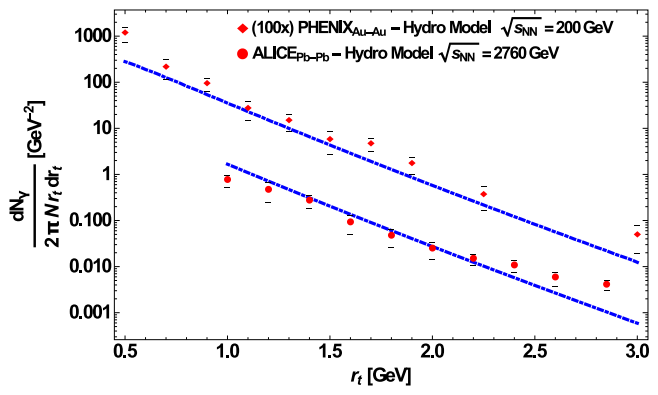

Figure 2. Experimental excess photon yield with respect to the hydro calculation of Ref. [13] compared to our calculation for the centrality class $0-20 \%$. The upper set corresponds to PHENIX data (multiplied by 100) and the lower set to ALICE data.

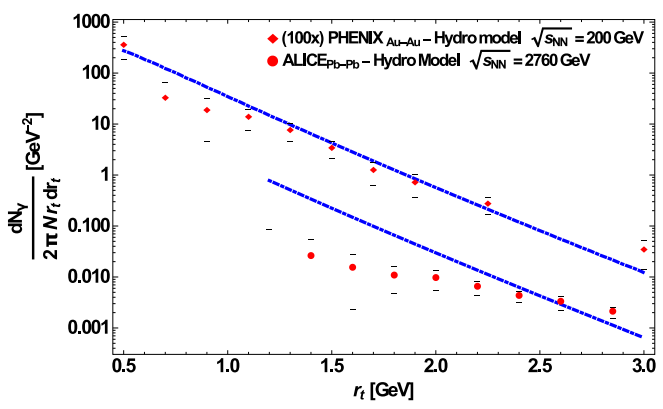

Figure 3. Experimental excess photon yield with respect to the hydro calculation of Ref. [13] compared to our calculation for the centrality class $20-40 \%$. The upper set corresponds to PHENIX data (multiplied by 100) and the lower set to ALICE data.

where $\mathcal{V} \mathcal{T}$ represents the space-time volume of the reaction coming from squaring $(2 \pi)^{4} \delta(r-v-u)$ and we have included the sum over the two light flavors $f=u, d$. The odd-looking factor $6256 / 2187 \sim 2.86$ is obtained from the longitudinal piece of the matrix element squared after collecting the coefficients of the contraction of the polynomial in the components of $u_{\|}$and $v_{\|}$. Notice that after the approximations made to compute Eq. (9), the dependence on $r_{\perp}$ of Eq. (11) comes exclusively from the transverse piece of the matrix element, Eq. (8).

The invariant photon yield is obtained by integrating over the corresponding phase space weighed with the thermal distribution, namely

$$
\frac{r_{0} d N}{d^{3} r}=\frac{1}{2(2 \pi)^{3}} \int \frac{d^{3} u}{2 u_{0}(2 \pi)^{3}} \int \frac{d^{3} v}{2 v_{0}(2 \pi)^{3}} \sum_{\operatorname{pol}_{, f}}|\mathcal{M}|^{2} n\left(u_{0}\right) n\left(v_{0}\right),
$$

where $n(E)=1 /\left[\exp \sqrt{E^{2}+m_{g}^{2}} / T-1\right]$ is the Bose-Einstein distribution and $T$ is the temperature.

The number of photons per unit momentum transverse to the beam axis, integrated over the full azimuthal angle, and around mid-rapidity, is explicitly given by

$$
\frac{d N}{d r_{t}}=C\left[\left(\frac{1}{3}\right)^{4} e^{-\frac{y_{0}^{2} r_{t}^{2}}{e B}}+\left(\frac{2}{3}\right)^{4} e^{-\frac{y_{0}^{2} r_{t}^{2}}{e B}}\right] I\left(\frac{r_{t}}{T} ; \sqrt{\frac{2}{3} g}\right)
$$


where we have evaluated the distribution at $y=y_{0}=0.5$, given that the rapidity interval $\Delta y \sim 1$ is centered around mid-rapidity. We have also defined

$$
C=\frac{g^{4} T e^{4} B^{2}}{8(2 \pi)^{7}}\left(\frac{6256}{2187}\right)\left(\frac{\mathcal{V} \mathcal{T}}{m_{g}^{2}}\right)
$$

and

$$
I(z ; \lambda) \equiv \int_{0}^{z} \frac{d x x^{2} n\left(\sqrt{(z+x)^{2}-(2 x)^{2}}\right) n(x)}{\left(\sqrt{x^{2}+\lambda^{2}}\right)\left(\sqrt{(z+x)^{2}-(2 x)^{2}+\lambda^{2}}\right)} .
$$

We have also used that in QCD with two flavors, $m_{g}^{2}=(2 / 3) g^{2} T^{2}$. The yield given by Eq. (13) can be properly called thermal because its computation assumes that the gluons are thermally distributed in phase space.

In order to be able to compare to ether data or models that do not consider photon emission induced by magnetic fields, we compute the yield normalized to the number of events. This is given by

$$
\frac{1 d N}{2 \pi N r_{t} d r_{t}}=\frac{\left[\left(\frac{1}{3}\right)^{4} e^{-2 \frac{y_{0}^{2} r_{t}^{2}}{e B}}+\left(\frac{2}{3}\right)^{4} e^{-\frac{y_{0}^{2} r_{t}^{2}}{e B}}\right] \frac{I\left(r_{t} / T\right)}{2 \pi}}{\sqrt{3 \pi e B / 2}\left[\left(\frac{2}{3}\right)^{9 / 2}+\left(\frac{1}{3}\right)^{9 / 2}\right] \int_{0}^{\infty} d r_{t} I\left(r_{t} / T\right)},
$$

where the factor $\sqrt{3 \pi e B / 2}$ in the denominator comes from the integration over rapidity. Notice that, despite having approximated $r_{\perp} \simeq 0$ for the calculation of the longitudinal matrix element, the normalization $N$ is computed extending the integration range over $r_{t}$ up to $+\infty$. This is a valid approximation, given that the main behavior of the rate is dictated by the exponential fall off in this variable coming from the transverse part of the matrix element squared. The normalized distribution is independent of the space-time region of the reaction. The impact parameter dependence is due to the dependence on the field intensity. The photon transverse yield given by Eq. (16) is an excess yield that should be added to calculations that do not consider magnetic field effects for photon emission. In order to compare with experimental data we first proceed to use appropriate values for the temperature, the coupling $g$, and the magnetic field strength. We take $g=1$ (the results turn out to be only marginally sensitive to the value of $g$ ). The variation of the field intensity with time and impact parameter for RHIC and LHC energies is taken from Ref. [19]. We chose one of the largest values of $e B$ which for RHIC, $\sqrt{s_{N N}}=200 \mathrm{GeV}$, correspond to $0.5 \times 10^{4}<e B /(\mathrm{MeV})^{2}<10^{5}$ and for LHC, $\sqrt{s_{N N}}=2.76$ $\mathrm{TeV}, e B /(\mathrm{MeV})^{2} \simeq 10^{4}$, with small variations coming from a slight dependence on the impact parameter. To relate the centrality class of the collision with the impact parameter we follow the geometrical model of Ref. [20]. Figures 2 and 3 show our results compared to the experimental excess photon yield with respect to one recent hydrodynamical calculation [13]. The latter has been shown to approach the description of the experimental data within the lowest part of the uncertainties. Figure 2 (Fig. 3) shows a comparison with the centrality class case $0-20 \%(20-40 \%)$. In each graph the upper set corresponds to PHENIX (multiplied by 100) and the lower to ALICE data. Notice that even with the ballpark choices of the parameters involved, our calculation provides a very good description of the excess photons. For the case of ALICE $20-40 \%$ our calculation overshoots the data. This can be due to the fact that the hydrodynamic calculation [13] which we use as the reference to compute the excess also overshoots the data, for this centrality class at low $p_{t}$.

In conclusion we have computed the matrix element squared for photon production from th gluon fusion process in the presence of a magnetic field in the context of heavy-ion collisions. Assuming that at very early times, the gluon population is thermal, we have used this matrix element to compute 
the thermal photon spectrum. For this purpose we have used a simple scenario using conservative values for the temperature and strength of the magnetic field. We have compared this photon rate with experimental data subtracting an state of the art calculation of the photon yield. The excess photon yield coming from the magnetic field effect shows a good agreement with the above. A more detailed analysis including up to date estimates of the the evolution of the magnetic field [21], with a less stringent set of assumptions and a quantification of the magnetic field induces asymmetry, is being carried out and will be reported elsewhere.

The authors acknowledge useful conversations with M. E. Tejeda-Yeomans. Support for this work has been received in part from PAPIIT-UNAM under grant number IN101515, by CONACyT under grant number 256494 and by the National Research Foundation (South Africa), and the Harry Oppenheimer Memorial Trust OMT Ref. 20242/02.

\section{References}

[1] I. Arsene et al. (BRAHMS Collaboration), Nucl. Phys. A757, 1 (2005); B. B. Back et al., Nucl. Phys. A 757, 28 (2005); J. Adams et al. (STAR Collaboration), Nucl. Phys. A757, 102 (2005); K. Adcox et al. (PHENIX Collaboration), Nucl. Phys. A757, 184 (2005); P. Jacobs and X. N. Wang, Prog. Part. Nucl. Phys. 54, 443 (2005).

[2] F. Becattini, J. Phys. Conf. Ser. 527, 012012 (2014) and references therein.

[3] D. E. Kharzeev, L. D. McLerran and H. J. Warringa, Nucl. Phys. A 803, 227 (2008).

[4] V. Skokov, A.Y. Illarionov, V. Toneev, Int. J. Mod. Phys. A 24, 5925 (2009).

[5] V. Voronyuk, V. D. Toneev, W. Cassing, E. L. Bratkovskaya, V. P. Konchakovski, S. A. Voloshin, Phys. Rev. C 83, 054911 (2011).

[6] A. Bzdak, V. Skokov, Phys. Lett. B 710, 171-174 (2012).

[7] G. Basar, D. E. Kharzeev, V. Skokov, Phys. Rev. Lett. 109, 202303 (2012).

[8] G. Basar, D. E. Kharzeev, E. V. Shuryak, Phys. Rev. C 90, 014905 (2014).

[9] K. Tuchin, Phys. Rev. C 91, 014902 (2015).

[10] G. Arciniega, P. Ortega, L. Patiño, J. High Energy Phys. 1404, 192 (2014); S. Y. Wu and D. L. Yang, J. High Energy Phys. 1308, 032 (2013) K. A. Mamo, J. High Energy Phys. 1308, 083 (2013).

[11] A. Adare et al. [PHENIX Collaboration], Phys. Rev. C 91, 064904 (2015); J. Adam et al. [ALICE Collaboration], Phys. Lett. B 754, 235 (2016).

[12] A. Adare et al. [PHENIX Collaboration], e-Print: arXiv:1509.07758 [nucl-ex]; D. Lohner, et al. (ALICE Collaboration), e-Print: arXiv:1212.3995 [nucl-ex].

[13] J.-F. Paquet, C. Shen, G. S. Denicol, M. Luzum, B. Schenke, S. Jeon, C. Gale, ePrint:arXiv:1509.06738 [hep-ph].

[14] H. van Hees, M. He, R. Rapp, Nucl. Phys. A 933, 256-271 (2015).

[15] O. Linnyk, V. Konchakovski, T. Steinert, W. Cassing, E. L. Bratkovskaya, Phys. Rev. C 92, 054914 (2015).

[16] For a recent review see C. Shen, e-Print: arXiv:1601.02563 [nucl-th].

[17] A. Ayala. J. D. Castaño-Yepes, C. A. Dominguez, L. A. Hernandez, arXiv:1604.02713 [hep-ph].

[18] J. Schwinger, Phys. Rev. 82, 664 (1951).

[19] Y. Zhong, C.-B. Yang, X. Cai, S.-Q. Feng, Adv. High Energy Phys. 2014, 193039 (2014).

[20] W. Broniowski, W. Florkowski, Phys. Rev. C 65, 024905 (2002).

[21] L. McLerran and V. Skokov, Nucl. Phys. A 929, 184-190 (2014); V. Voronyuk, V. D. Toneev, W. Cassing, E. L. Bratkovskaya, V. P. Konchakovski, and S. A. Voloshin, Phys. Rev. C 83, 054911. 